\title{
Game-theoretic insights into the role of environmentalism and social- ecological relevance: A cognitive model of resource consumption
}

\author{
Talha Manzoor $^{1,2}$, Elena Rovenskaya ${ }^{3,4} \&$ Abubakr Muhammad ${ }^{1,2}$
}

\begin{abstract}
The environmental and social attitudes of consumers can greatly affect the consumption of common-pool resources by the whole society - as demonstrated by many experimental and theoretical studies. Rooting in the current knowledge of consumer psychology, and employing the game-theoretic modelling framework, we formalise the decision making process of individuals about their resource consumption levels depending on their level of environmental concern, and relative importance placed on social and ecological information. Our model demonstrates, in a stylized fashion, how profound preference to social information can help avert free-riding behaviour and result in globally stable resource consumption dynamics. This avoids the "Tragedy of the Commons", leading to affluence in the resource stock as well as in the individual consumption. Furthermore, we find that heterogeneity of the levels of individuals' environmentalism promotes free-riding, whereas heterogeneity in relative information preferences helps avoiding tragedies. Our analysis demonstrates that accounting for heterogeneity of consumers and their social relationships can yield additional insights regarding to what kind of societies may have better chances to ensure sustainable consumption of a natural resource.
\end{abstract}

Keywords: resource consumption; natural resource dynamics; social-comparison processes; tragedy of the commons; cooperation; heterogeneity

\section{Introduction}

Understanding and taking into account consumer behaviour in socio-ecological systems

${ }^{1}$ Department of Electrical Engineering, Lahore University of Management Sciences (LUMS), Pakistan

${ }^{2}$ Center for Water Informatics \& Technology (WIT), Lahore University of Management Sciences (LUMS), Pakistan

${ }^{3}$ International Institute for Applied Systems Analysis (IIASA), Laxenburg, Austria

${ }^{4}$ Faculty of Computational Mathematics and Cybernetics, Lomonosov Moscow State University, Moscow, Russia 
is of prime importance for designing an effective solution to phenomena, which are usually referred to as "The Tragedy of the Commons" (Hardin 1968). Social psychologists have carried out a wealth of research to identify factors driving the decision processes of individuals. For instance, Festinger (Festinger 1954) hypothesizes, that human beings are continuously driven to evaluate their decisions, and, in the absence of any objective non-social means, they do so by comparison with decisions of other individuals. Thus, if a consumer is uncertain about the state of a resource, she may make her decision based on the information gathered from other consumers. Simon postulates (Simon 1976), that individuals seldom optimize their outcomes over different available alternatives, mainly due to their limited cognitive resources, and instead act according to automated habitual behaviour. This implies, that although a consumer might be aware of the consequences of over-utilization of a natural resource, she may still do so according to habit, as long as the outcome is satisfying to her for the timebeing (assuming that there is enough of the resource to permit her over-utilization). These theoretical propositions have been supplemented by various laboratory and field experiments of common-pool resource settings. Samuelson et al. (Samuelson et al. 1984) conduct an experiment to observe how consumers respond to information on the overall consumption of the resource. They find that individual consumptions tend to increase over time, however there is little or no increase in consumption when the resource is being overused. Rutte et al. (Rutte, Wilke \& Messick 1987) demonstrate, through an experiment, that consumer behaviour is determined by whether the society or the environment is held responsible for the scarcity or abundance of resource. They conclude that when the environment is held responsible, consumers give ecological information more preference than social information, for evaluation of their consumption levels. Accordingly, when the society is held responsible, social 
information is given more preference than ecological information. Brucks and Mosler (Brucks \& Mosler 2011) conduct an experiment to find what type of information is important to consumers while making decisions on consumption. They observe, among other findings, that when the resource availability is low, the importance of the information regarding the situation of the resource increases, while the importance of the information regarding consumption of other individuals decreases.

Building on the aforementioned psychological research, scientists have proposed various computational models that simulate consumer behaviour during resource crises. Much of these models have been developed under the umbrella of "multi-agent simulations", or "agent-based modelling" (these terms are often used interchangeably), in which several heterogeneous individuals (agents) are programmed according to some psychological rules. Such a framework can be used to identify key behavioural elements that shape large-scale societal transitions. Deadman (Deadman 1999) presents one of the first agent-based models of the tragedy of the commons. Since then, various computational agent-based models of resource dilemmas have been developed - see, for instance, (Jager et al. 2000; Feuillette, Bousquet \& Le Goulven 2003; Jager \& Mosler 2007). These models differ in the principles used to simulate the behaviour of individual agents, as each group of authors, depending upon the posed research question, attempts to incorporate different psychological findings into the behaviour rules of agents. Bosquet and Le Page (Bousquet \& Le Page 2004) give an extensive review of the development of this field and early applications to ecosystem management.

Despite the power of computational agent-based models to reveal collective phenomena from individual interactions, they lack rigor, generality and elegance that is provided by mathematical models. Notable achievements on the latter front include, for example, Anderson (Anderson 1974) who describes the coupled dynamics of a 
regenerating commons and the physical capital, as a system of few ordinary differential equations, and uses calculus to show that the model exhibits Hardin's conclusions (Hardin 1968) explicitly. Anderies (Anderies 2000) applies bifurcation analysis to two separate models of resource exploitation: (1) the slash-and-burn agricultural system of the Tsembaga tribesmen of New Guinea, and (2) the exploitation of palm forests on Easter Island by the Polynesians. The analysis is used to study the long-term behaviour of both systems under different management/ behavioural regimes. The study suggests that successful institutional designs are highly site-specific and that a careful understanding of the "geometry" of the system is necessary for successful resource governance. Roopnarine (Roopnarine 2013) suggests simple differential equation models which separately illuminate three different aspects of the tragedy of the commons: (1) the compulsion of individual users to act based on the action of other users, (2) the opportunities and limitations imposed by the networked nature of consumers, and (3) mutualisms as solutions to tragedies. Thus, these mathematical models are able to demonstrate and explain a certain phenomenon in a clear and tractable fashion, where a major focus is usually placed on the dynamics of consumption. Although no objective function or decision variables are explicitly included in their formulation, they typically assume some notion of rationality prevalent in the consumers. However, the theoretical findings provided by social psychologists are seldom incorporated in these models.

The commons dilemma has also been studied extensively in the framework of game theory. In her seminal work, Ostrom states that all institutional arrangements that can be used to avert the commons dilemma are expressible as games in extensive form, and presents several such examples (Ostrom 1990; Ostrom, Gardner \& Walker 1994). While she expresses institutionalization for resource governance as a non-cooperative 
game, a large number of analysts have also used cooperative game theory to study environmental resource issues (see, for example, (Parrachino, Dinar \& Patrone 2006)). Ostrom's research has stimulated a vast amount of work, concerning use of the game theoretic framework for modelling strategic interactions between consumers of a natural resource (see for instance (Madani 2010; Cole \& Grossman 2010; Diekert 2012)). Similar to system dynamic models, game-theoretic models also assume a notion of rationality, which is made explicit by including objective functions and decision variables in the problem formulation. However, contrary to dynamical system models, they either do not take dynamics into account or do so in a simplified manner (as repeated games). More importantly, most game-theoretic models, either seek an optimal strategy of resource extraction, or seek to design models that yield strategies similar to those observed in real-world scenarios, rather than focusing on strategies that accurately depict the cognitive process of the consumers' decision making. We argue, that in order to increase the relevance of such models to real-world scenarios, it is necessary to incorporate the cognitive principles that govern consumer behaviour, as revealed by the psychological research.

In this paper, we present a dynamic model of natural resource consumption, taking into account the psychology of consumer behaviour in an open-access setting. We depart from the computational model by Mosler and Brucks (Mosler \& Brucks 2003) and put forward a stylized version that is based on the same psychological principles employed in the original model. All essential psychological variables have been maintained in our model, which include the scarcity thresholds as perceived by consumers, the extent to which the consumers hold nature (or society) responsible for the state of the resource, and the social value of the consumers. While the original model assumes unlimited growth of the resource, we assume standard logistic growth, 
which is more realistic. The logistic growth model and its variants are commonly used to model many renewable resources that saturate at a certain carrying capacity. Thus the resources which lie in the scope of our study include (but are not limited to) fisheries (Gordon 1954), forests (Fekedulegn, Mac Siúrtáin \& Colbert 1999), vegetation (Birch 1999), foliage (Werker \& Jaggard 1997), saffron (Torabi, Saadatkhah \& Soltani 2014), and so on. We do not, in this study, incorporate the effects of uncertainty in our model. Thus we assume, that perfect information is available to each consumer, regarding the resource quantity and the consumption of other individuals present in the system. While this is a strong and restrictive assumption, incorporating uncertainty is beyond the scope of this paper, as it would considerably increase the complexity of the model, and must be dealt with separately. Thus, we present the reformulation, which enables formal tractable mathematical treatment of the model. We carry out the steady-state analysis, consider our system in equilibrium and employ the game-theoretic framework to study what conditions lead to the commons problem. Namely, we introduce a non-cooperative continuous-kernel game to analyse the rational decisions of consumers for different combinations of key model parameters describing the resource dynamics and the society. Furthermore, we define a notion of "tragedy" in the commons game, based on the distance between the Nash equilibrium and the Pareto optimum. We then use exhaustive numeric simulations to reveal such trends in the system parameters, which are helpful for decreasing "tragicness" and are also beneficial to the resource stock.

\section{Methods}

Our starting point is the computational consumer behaviour model of Mosler and Brucks (Mosler \& Brucks 2003), which we stylize by formalizing it into a mathematical form. The resource is supposed to regenerate according to the classical logistic growth 
model (Perman 2003). To begin with, we consider a society consisting of two consumer groups characterized by significantly different psychological characteristics. Within the groups these characteristics are similar, thus the groups are assumed to be internally homogeneous. This phenomenon of social polarization has been well studied in the past and also observed in real-world settings (Chakravarty 2015; Zwiers, Kleinhans \& Van Ham 2015). Under this assumption it is possible to describe their behaviour through two aggregated equations.

Each group is assumed to have open-access to a resource, which it consumes by exerting some effort. The dynamics of the group's effort are modelled based on our understanding of the cognitive process depending on certain psychological characteristics of the group. These characteristics depict: (1) the environmentalism of the group (the perceived benchmark quantity that the group uses to evaluate whether the resource is in abundance or in scarcity), (2) the social values of the group (how cooperative is the group), and (3) causal attributions of the group (to what extent does the group attribute the current condition of the resource to nature-induced reasons, as compared to society-induced reasons).

\section{$2.1 \quad$ Resource Dynamics}

Consider a society with two consumer groups, each having open-access to a single renewable resource. We assume that the resource quantity $R(t)$, available for consumption by the society at time $t$, has an associated growth function, which is logistic in nature. We further assume, that in the absence of consumption, $R(t)$ increases over time at an intrinsic (positive, constant) growth rate $r$ and saturates at the given carrying capacity $R_{\max }$. The two groups, each identified by its respective index $i=1,2$, exert effort $e_{i}(t)$ respectively to consume the resource. The resource 
growth is, therefore, given by

$$
\frac{d R(t)}{d t}=r\left(1-\frac{R(t)}{R_{\max }}\right) R(t)-\left(e_{1}(t)+e_{2}(t)\right) R(t)
$$

where each group's harvest of the resource equals $e_{i}(t) R(t)$. Equation (1) is equivalent to the standard Gordon-Schaefer model (Gordon 1954), with the catch coefficient set to unity.

\subsection{Consumption Dynamics}

Here we define the dynamics of the effort $e_{i}(t)$ over time. We assume that the change in consumption of each group, is based on their weighing of two different factors, one pertaining to information about the resource quantity (the ecological factor), and second pertaining to information about the use of others (the social factor) (Brucks \& Mosler 2011). Which factor gains precedence over the other in the cognitive process of harvesting decisions depends on the individual characteristics of each group, depicted here through multiple psychological variables. The final change in consumption is the sum of the ecological and social impacts, where we define the impact as the product of the corresponding factor and its weight. Thus the change in effort is given by

$$
\frac{d e_{i}(t)}{d t}=\text { ecological weight } \times \text { ecological factor }+ \text { social weight } \times \text { social factor }
$$

In what follows, we specify the ecological and social factors, and the psychological variables that determine the relative weighing of these factors to determine the change in effort $e_{i}(t)$. The final Equation is given by (2).

Namely, Rutte et al. conclude from their study (Rutte, Wilke \& Messick 1987) that consumers harvest more from the resource in abundance than in scarcity. They further observe that harvests for each individual tend to increase over time, except when 
the resource is scarce, in which case it decreases. We implement this effect in our model by comparing the current resource stock with a perceived-by-the-consumer "scarcity threshold", denoted as $R_{i} \in\left[0, R_{\max }\right]$. Thus we define the ecological factor for $i$ as the difference $R(t)-R_{i}$. A positive ecological factor represents an abundant state of the resource, whereas a negative factor represents a scarce state. A scarce resource in the perception of group $i$ results in a decrease in consumption, whereas an abundant resource results in an increase. Note that that abundance or scarcity represent only the beliefs of the consumers and may or may not depict the objective state of the resource. While Mosler and Brucks (Mosler \& Brucks 2003) assume the scarcity threshold to be constant for the entire population, we generalize by allowing it to be different for each consumer (thereby ascribing more influence of the heterogeneity of consumers). Indeed, consumers with different characteristics (age, sex, social class, political orientation, etc.) also tend to have different levels of environmental concern (Van Liere \& Dunlap 1980).

In the same study, Rutte et al. (Rutte, Wilke \& Messick 1987) show that consumer behaviour differs, depending on the extent, to which they attribute scarcity of the resource to nature-induced reasons relative to society-induced reasons. For example in the case of a forest, nature-induced reasons may include less rainfall, volcanic eruption, and so on. Society-induced reasons may include extensive overuse by the consumers, pollution, and other practices that are harmful to the forest. We represent this attribution of $i$ as $a_{i} \geq 0$; where $a_{i}=0$ represents a consumer group, which associates the scarcity of the resource entirely with society, with increasing values of $a_{i}$ representing increasing association of the resource scarcity to nature. As suggested by the aforementioned study, the consumers that attribute the condition of the resource more to nature, tend to give ecological information more importance. Thus we define the ecological weight to be equal to the attribution $a_{i}$. The ecological impact is therefore 
given by $a_{i}\left(R(t)-R_{i}\right)$, which means that a consumer group, which attributes the condition of the resource more to nature (thus having a high value of $a_{i}$ ) will manifest a higher ecological impact, than a group, which attributes the condition of the resource to society (having a low value of $a_{i}$ ).

In his theory of social comparison processes, Festinger (Festinger 1954) postulates that people are less attracted to situations, where others are very divergent from them, than to situations, where others are close to them regarding both abilities and opinions. Therefore, we define the social factor by the difference $e_{j}(t)-e_{i}(t)$, where $i, j=1,2$ and $i \neq j$. Thus the social factor is a measure of equality, with a low magnitude representing a society with equal consumption (dictating a lesser change in consumption) and a high magnitude representing unequal consumption (dictating a greater change in consumption). Furthermore, a negative social factor represents a higher consumption of the target group relative to the other group (dictating a decrease in the target group's consumption) and a positive social factor represents a relatively lower consumption of the target group (dictating an increase in consumption).

The social value of consumer group $i$ is represented by $s_{i} \geq 0$. Here $s_{i}=0$ represents an extremely non-cooperative individual, with increasing values of $s_{i}$ representing an increasingly cooperative individual. A consumer with higher social value will weigh equality more heavily than a consumer with lower social value. The influence of the social value of consumer $i$, represented by $s_{i} \geq 0$, is based on the assumption that cooperative individuals are more concerned with maximizing equality and respond with anger to violations in equality regardless of the effect on their own outcomes (Van Lange et al. 2013). Thus the social impact is given by the product $s_{i}\left(e_{j}(t)-e_{i}(t)\right)$. 
Thus in our assumed society of two consumer groups, the following equation gives the change in effort of group $i$ as a function of its individual characteristics, the current stock level and the effort of the other group

$$
\frac{d e_{i}(t)}{d t}=a_{i}\left(R(t)-R_{i}\right)+s_{i}\left(e_{j}(t)-e_{i}(t)\right)
$$

where $i, j=1,2$ and $i \neq j$. The first term on the right hand side of (2) represents the weighted ecological factor, where $\left(R(t)-R_{i}\right)$ is the difference between the perceived optimum and actual level of the stock, which is weighed by the attribution $a_{i}$. The second term on the right hand side of (2) represents the weighted social factor, where $\left(e_{j}(t)-e_{i}(t)\right)$ is the difference in effort between both groups, which is weighed by the social value $s_{i}$. It is important to note that the positive values of $s_{i}$ assumed here, imply that both groups are socially connected to each other, and have knowledge about each other's consumption.

\subsection{Coupled socio-ecological system}

Together (1) and (2) describe the overall dynamics of the considered socio-ecological system of the two-group society consuming an open-access resource. In what follows, we undertake some additional transformations, after which the model is able to capture the first principles more accurately, and the new variables have clearer interpretations in terms of the underlying theory. The transformations also reduce the overall dimensionality of the parameter space.

Let $x(t)$ be the quantity of the resource relative to the carrying capacity $R_{\max }$ of the environment and $y_{i}(t)$ be $i$ 's effort relative to the intrinsic growth rate $r: x(t)=$ $R(t) / R_{\max }$ and $y_{i}(t)=e_{i}(t) / r$. Define $\rho_{i}=R_{i} / R_{\max }$ as $i$ 's scarcity threshold level relative to $R_{\max }$. Next define $\tau=r t$ as the new, non-dimensional time scale. We can 
now express the system as follows

$$
\begin{gathered}
\dot{x}=(1-x) x-\left(y_{1}+y_{2}\right) x, \\
\dot{y}_{1}=b_{1}\left(\alpha_{1}\left(x-\rho_{1}\right)+v_{1}\left(y_{2}-y_{1}\right)\right), \\
\dot{y}_{2}=b_{2}\left(\alpha_{2}\left(x-\rho_{2}\right)+v_{2}\left(y_{1}-y_{2}\right)\right),
\end{gathered}
$$

where $b_{i}=\frac{\left(a_{i} R_{\max }+r s_{i}\right)}{r^{2}}, \alpha_{i}=\frac{a_{i} R_{\max }}{a_{i} R_{\max }+r s_{i}}, v_{i}=\frac{r s_{i}}{a_{i} R_{\max }+r s_{i}}$ and the over-dot represents the derivative with respect to $\tau$. Although the original weights $a_{i}$ and $s_{i}$ have different dimensions and thus are incomparable, the new weights $\alpha_{i}$ and $v_{i}$ become dimensionless and can be compared. Furthermore, $\alpha_{i}+v_{i}=1$, which means that $\alpha_{i}$ and $v_{i}$ are complements of each other. This reflects the bipolarity in social and physical dimensions as described by the theory underlying the original model (Mosler \& Brucks 2003). Thus $\alpha_{i}$ can be interpreted as the relevance that $i$ assigns to ecological information and $v_{i}$ can be interpreted as the relevance that $i$ assigns to social information. The state variables $x, y_{i}$ and parameters $\rho_{i}, b_{i}$ also become dimensionless. Henceforth, we call $\alpha_{i}$ and $v_{i}$ the ecological and social relevance of $i$ respectively, and $\rho_{i}$ the environmentalism of $i$, where $\rho_{i}=1$ represents an extremely environmental group and $\rho_{i}=0$ represents an extremely non-environmental group. Here $b_{i}$ can be interpreted as the overall susceptibility (Friedkin 2006) of group $i$ to change in its consumption. All subsequent analysis in this paper will be carried out on model (3).

Note that the dynamics given by (3) allow the possibility of negative values for $y_{1}(\tau)$ and $y_{2}(\tau)$, which means that an interpretation of negative effort is required. As defined by (Perman 2003), all the different dimensions of harvesting activity can be aggregated into one magnitude called effort. Negative effort means any effort exerted for the sustenance of the resource. It is not limited to growing trees or breeding fish, but 
can also include, for fisheries, the restriction of fishing gear, closed area management, awareness programs and so on (Worm et al. 2009). Similarly for forests this can include restoration of soil fertility, preservation of remnant vegetation and promoting community forest enterprises (Chazdon 2003; Bray et al. 2003). One can imagine similar realizations of all resources that fall under the scope of this study.

Natural resources have been essential inputs to economic development, which is considered necessary to overcome world poverty. It has been argued for decades now that "development" cannot imply infinite economic growth, as it has obvious restrictions due to the finite limits of the environment, which houses our economic system (Perman 2003; Meadows et al. 1972). Many influential economists have championed the concept of a steady-state economy, where the question is not that of how to achieve maximum growth, but of how to realize the most attractive equilibrium. In the past, there has been extensive debate on the viability and usefulness for the practice of the concept of the steady-state economy. Some recent studies suggest to interpret the economy's steady-state as an "unattainable-goal" (Kerschner 2010) and to view its analysis as an efficient tool, which is useful to guide the design of the long-term policy for real-world economies. This gives special importance to examining the steady states of mathematical models of economic growth and resource consumption, for providing long-term strategies especially in the context of sustainability. In this spirit, we analyse the equilibrium of (3), which is found out to be unique and given as

$$
\begin{aligned}
\bar{x} & =\frac{\alpha_{2} \rho_{2} v_{1}+\alpha_{1} \rho_{1} v_{2}}{\alpha_{2} v_{1}+\alpha_{1} v_{2}} \\
\bar{y}_{1} & =\frac{\left(1-\rho_{2}\right) \alpha_{2} v_{1}+\left(1-\rho_{1}\right) \alpha_{1} v_{2}-\left(\rho_{1}-\rho_{2}\right) \alpha_{1} \alpha_{2}}{2\left(\alpha_{2} v_{1}+\alpha_{1} v_{2}\right)}, \\
\bar{y}_{2} & =\frac{\left(1-\rho_{2}\right) \alpha_{2} v_{1}+\left(1-\rho_{1}\right) \alpha_{1} v_{2}-\left(\rho_{2}-\rho_{1}\right) \alpha_{1} \alpha_{2}}{2\left(\alpha_{2} v_{1}+\alpha_{1} v_{2}\right)}
\end{aligned}
$$


Here $\bar{x}$ turns out to lie between 0 and 1 for all admissible parameter values, which ensures a non-negative resource stock that does not exceed the carrying capacity. The efforts $\bar{y}_{i}$ can be either both positive, or have different signs, which is discussed in Section 3.

If equilibrium (4) appears to be stable, in the long-term, the society converges to a stable resource stock and stable consumption rate regardless of the initial state of the system. As shown in the Appendix, the equilibrium is guaranteed to be stable except for very small values of $v_{i}$ and $\rho_{i}$ which corresponds to a society that is extremely nonenvironmental and has extremely low social value.

\subsection{Game-theoretic formulation}

The tragedy of the commons can be seen as one particular instance of the more general problem of eliciting cooperation between individuals, when there exists a temptation for individuals to defect. Hardin (Hardin 1968) was one of the first who proposed to employ centralized mechanisms to coerce a society in order to achieve collectively optimal outcomes in such situations. He further asserted that any decentralized mechanism, regulated from within the society, must necessarily fail in achieving those outcomes. This assertion was challenged by Ostrom (Ostrom 1990), who reported several real-world examples of successful institutions, conceived and regulated by the communities themselves, and used a game theoretic framework to conceive a new theory of collective action. Typical game theoretic formalizations, which have been used to depict tragedies, include the prisoner's dilemma, the assurance game and the snowdrift game. These games have been studied in both discrete and continuous action spaces (Doebeli \& Hauert 2005). 
Here we formalize the socio-ecological system in (3) as a static, one-shot twoplayer game, in which two consumer groups (players) have access to a single common good (natural resource). Each group decides on the level of environmentalism $\rho_{i} \in$ $[0,1]$ it manifests, which in turn determines the steady state effort it exerts in consuming the resource according to (4). Thus the set of alternatives available to each player is a continuum, which constitutes a continuous-kernel game. The amount of resource consumed by a group at the steady state is assumed to be its payoff i.e., the payoff function is given by $\pi_{i}\left(\rho_{i}, \rho_{j}\right)=\bar{x} \bar{y}_{i}$, where $i, j=1,2$ and $i \neq j$.

We now derive the Nash equilibrium of the constructed game. In this model, the Nash equilibrium represents a state of society, in which no single group has an incentive (in terms of increasing its payoff) to consume more of the resource (by means of changing its environmentalism) given that the other group adheres to its current level of environmentalism. Thus if both groups consume as prescribed by the Nash equilibrium, no group is expected to deviate from their consumption levels. It is defined more technically as follows. First consider the "best response" of a group, which is the level of environmentalism that maximizes its payoff, given an environmentalism of the other group. The Nash equilibrium is then given by the intersection of two best responses $\rho_{1}^{\#}\left(\rho_{2}\right), \rho_{2}^{\#}\left(\rho_{1}\right)$ such that each strategy is a best response to the other. We find this to be given by

$$
\rho_{i}^{\#}=\frac{v_{i}\left(3 v_{j}-v_{i}-2 v_{i} v_{j}\right)}{\left(1-v_{i}\right)\left(v_{i}+v_{j}+2 v_{i} v_{j}\right)} \quad(i, j=1,2, i \neq j)
$$

Note that the social relevance $v_{i}$ is considered as a given parameter here, and not as a decision variable. We find (see appendix) that including $v_{i}$ in the strategy set results in an infinite number of Nash equilibria defined exactly in the form shown above; so 
treating $v_{i}$ as exogenous parameters simplifies the further analysis and seems not to affect the findings substantially.

A property inherent to any representation of the tragedy of the commons is that individually rational behaviour leads to outcomes that are collectively suboptimal. We thus compare the environmentalism of each group at the Nash equilibrium with one that maximizes the utilitarian welfare function of total consumption $\pi_{1}\left(\rho_{1}, \rho_{2}\right)+\pi_{2}\left(\rho_{2}, \rho_{1}\right)$. We find that the latter optimum $\left(\rho_{1}^{*}, \rho_{2}^{*}\right)$ is not unique, and is given by the following curve

$$
2 v_{2}\left(1-v_{1}\right) \rho_{1}^{*}+2 v_{1}\left(1-v_{2}\right) \rho_{2}^{*}-v_{1}\left(1-v_{2}\right)-v_{2}\left(1-v_{1}\right)=0,
$$

with the Nash equilibrium situated at some distance from it (naturally determined by parameters $v_{1}$ and $\left.v_{2}\right)$. We call this distance the tragicness of the consumption game, which, more specifically, is defined here as the length of the shortest line joining the Nash equilibrium and a point on the optimal curve (see Figure 1). Thus in a non-tragic game, the Nash equilibrium would lie exactly on the welfare optimal curve (resulting in tragicness equal to zero). All other games in which the Nash equilibrium does not lie on this curve are classified as tragic games, where the magnitude of the distance between the Nash equilibrium and the Pareto-optimal solutions is quantified through their tragicness. The concept of tragicness, which we introduce here, resembles the "price of anarchy" (Koutsoupias \& Papadimitriou 2009), which is a ratio between the cost of the worst possible Nash equilibrium and the optimum of a social welfare function as measure of effectiveness of the system. Another similar concept is the "price of stability" (Anshelevich et al. 2008), which is a ratio between the cost of the best possible Nash equilibrium and the optimal solution. Both concepts have been applied widely in computer network design. 


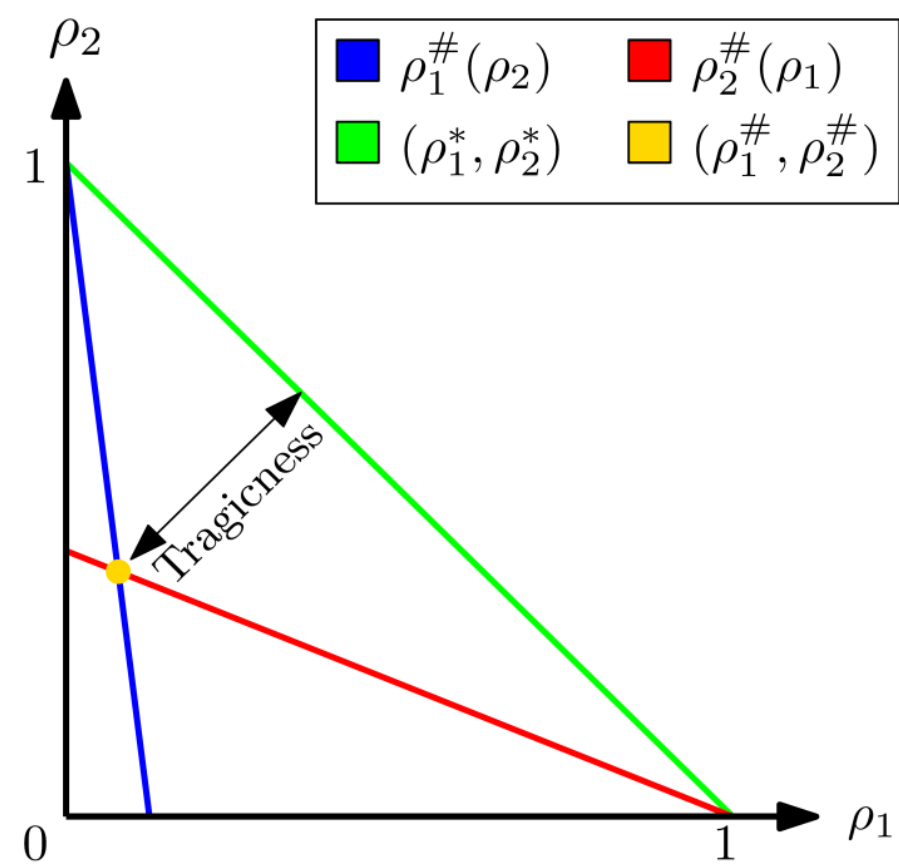

Figure 1: An illustration of the definition of the game tragicness.

\section{Results}

In Section 2.3 we presented the steady state of the socio-ecological system (3). Based on the values of parameters $v_{i}$ and $\rho_{i}$, two types of steady state behaviour may occur either both of the groups consume at a positive rate or one consumes at a negative rate, i.e., contributes to the resource, while the other free-rides and consumes at a positive rate. After presenting the comparative statics results for system (3) in Section 3.1, we attempt to answer the question of how to limit this free-riding behaviour by analysing the positivity of the steady state across the parameter space. This analysis is conducted in Section 3.2. The consumer game presented in Section 2.4 illustrates strategic interactions between two consumer groups. In Section 3.3 below, we explore how the game characteristics, such as the tragicness, steady-state resource stock, individual consumption and equity are affected by the model parameters, most notably, by the social relevance $v_{i}$. On this basis we deduce favourable conditions for successfully sustaining the natural resource, while also maintaining an acceptable level of 
consumption.

\subsection{Comparative statics}

Here we inspect how the magnitudes of the steady-state resource quantity $\bar{x}$, and the steady-state consumption efforts $\bar{y}_{1}$ and $\bar{y}_{2}$ change in response to changes in the parameters $\rho_{1}, \rho_{2}, v_{1}$ and $v_{2}$. We do this by conducting a comparative statics analysis (Perman 2003), which is undertaken by obtaining the first-order partial derivatives of each of the three equations in (4), and determining whether or not an unambiguous sign can be attached to each partial derivative. Table 1 shows the results for the analysis. A plus sign means that the derivative is positive, a minus sign means that the derivative is negative, ' 0 ' means that the derivative is zero and a '?' means that no sign can be assigned unambiguously to the derivative.

Table 1: Comparative statics results for system (3).

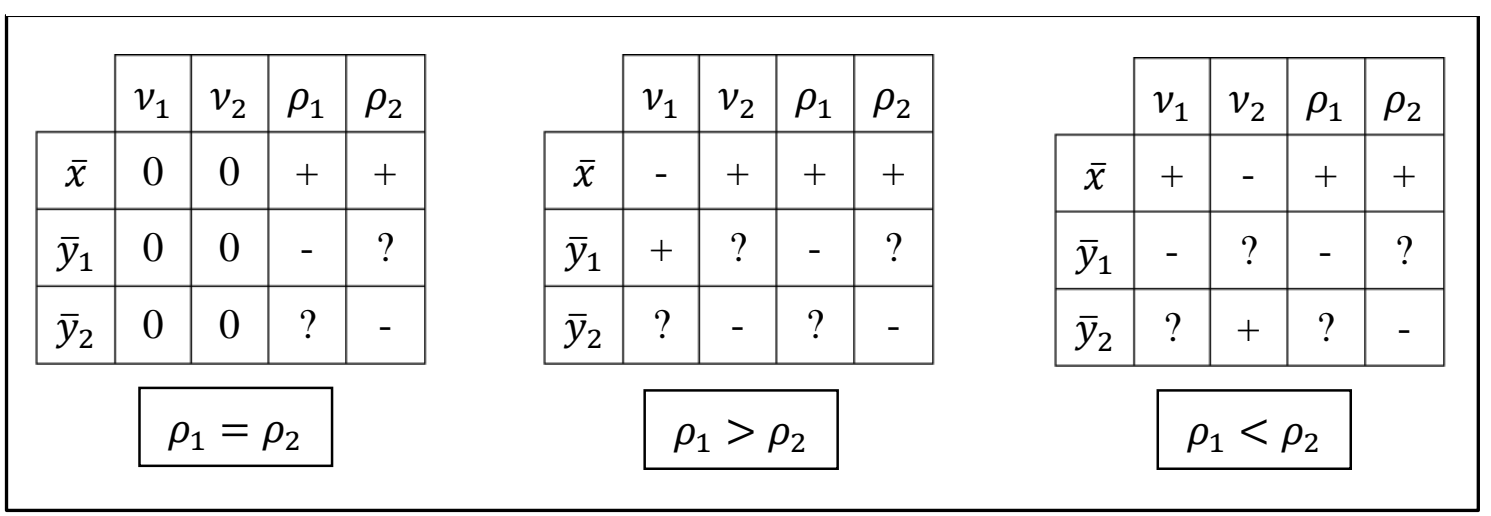

From Table 1, we see that an increase (decrease) in the levels of environmentalism $\rho_{1}$ and $\rho_{2}$, always increases (decreases) the steady-state resource stock $\bar{x}$. Thus higher environmentalism results in a larger resource stock at steady-state. In contrast, an increase in the environmentalism $\rho_{i}$ of individual $i$, decreases the steadystate consumption $\bar{y}_{i}$ of $i$, which means that environmental individuals consume less at 
steady-state. It can also be noted that the effect of $\rho_{i}$ on $\bar{y}_{j}$, where $i \neq j$, is ambiguous and not much can be said by simply observing the first-order partial derivatives.

The effect of the social relevances $v_{1}, v_{2}$ on the steady state varies according to the relative magnitudes of the environmentalisms $\rho_{1}, \rho_{2}$. When both groups are equally environmental $\left(\rho_{1}=\rho_{2}\right)$ then the social values do not affect the steady-state. When this is not the case, the social value of the relatively environmental (non-environmental) group has a negative (positive) effect on the steady-state resource stock. Furthermore, the social value of the relatively environmental (non-environmental) group has a positive (negative) effect on the group's own steady-state consumption effort. We see that the effects of the parameters on the relationship between the steady-state consumption efforts are not entirely revealed by this exercise, and require additional analysis to uncover. This is done in Section 3.2 below.

\subsection{Equilibrium and the free-riding phenomenon}

Ostrom's work (Ostrom 1990) reports several real-world examples of free-riding in open-access resource settings, which is a major cause of concern for the successful governance of such resources. In such settings, the free-riding phenomenon corresponds to situations where certain individuals (the free riders) benefit from the resource without contributing to its sustenance. System (3) captures this through two possible types of equilibrium. We label these two types as the "self-reliant" equilibrium and the "freeriding" equilibrium. The self-reliant equilibrium represents both consumer groups harvesting at a positive rate (both $\bar{y}_{1}$ and $\bar{y}_{2}$ of (4) are positive). The free-riding equilibrium represents one of the groups harvesting at a positive rate and the other harvesting at a negative rate (one of $\bar{y}_{1}$ and $\bar{y}_{2}$ is positive, while the other negative). Thus one of the groups exerts effort into increasing the resource quantity, while the 
other free-rides and enjoys a positive consumption.

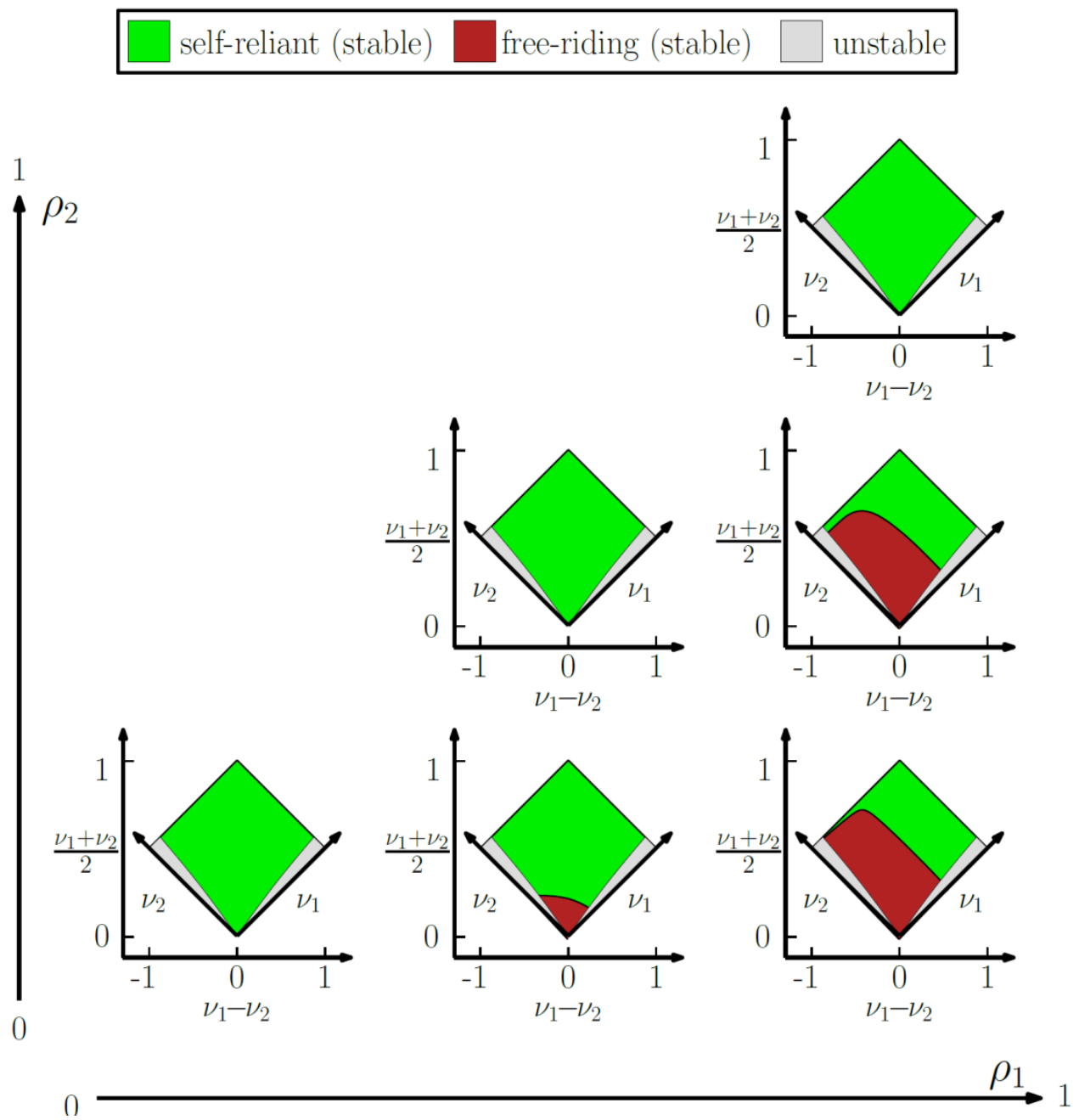

Figure 2: Positivity of the equilibrium in the $v_{1}, v_{2}$ plane for different values of $\rho_{1}$ and $\rho_{2}$. The plots are symmetric in $\rho_{1}$ and $\rho_{2}$ and so the missing plots are simply a reflection of the existing plots about the symmetric axis. The freeriding equilibrium corresponds to $\bar{y}_{1}<0<\bar{y}_{2}$.

An exhaustive simulation of the equilibrium consumption values in the parameter space is shown in Figure 2. We see that free-riding is excluded only when both groups have the same environmentalism level $\rho_{i}$. When groups are different in what concerns their environmentalism, the equilibrium is self-reliant if the group corresponding to the higher level of environmentalism has a social relevance beyond a 
certain level. This level can be interpreted as the reluctance of the relatively environmental group to subsidize the consumption of the relatively non-environmental group. The reluctance increases with increasing levels of social relevance, and indirectly with the level of cooperativeness of the environmental group. Thus the more cooperative a group becomes (consequently possessing a higher social relevance) the more reluctant it is to subsidize the non-environmental group. This is consistent with the postulate that cooperative individuals promote equality, and favour outcomes which maximize joint benefits (contrasted with altruistic individuals, who willingly sacrifice their own benefit in order to maximize the benefit of others). Interestingly, it has been observed that cooperative individuals are also likely to have high levels of environmentalism and vice-versa (De Groot \& Steg 2010; Gärling et al. 2003), which in the context of our model implies that it is really the social relevance of the groups that determines whether they end up in a self-reliant or free-riding equilibrium.

\subsection{Social-ecological relevance in the consumption game}

Here we present insights gained by observing how the characteristics of the consumption game depend on the model parameters and what it entails.

\subsubsection{The anti-tragic role of social relevance}

Cooperative behaviour plays a key role in avoiding tragedies and increasing the steady state resource stock. In our model, this effect can be observed through Figure 3(a)-(c). Figure 3(a) shows that the tragicness of the consumption game decreases as we move away from the origin along any of the $v_{1}$ or $v_{2}$ axes. Furthermore, the tragicness declines with an increase in the average (over two groups) level of social relevance. Declining of the tragicness can indeed be seen as growing of coordination and cooperation (through increasing social relevance) between two groups. Games, which are less tragic, 
correspond to affluence in the steady state resource stock, which in-turn also allows for a higher resource consumption at steady state. Thus the same trends can be observed for the steady state resource stock and total consumption rate in Figure 3(b) and (c) respectively. This shows that high social relevance not only decreases the game's tragicness, but also favours higher steady state resource stocks and consumption rates.

In the limit case of $v_{1}=v_{2}=1$, zero tragicness is achieved over the infinitely many equilibria, and it is interesting to note that in this most affluent case, the maximum resource stock $\bar{x}=0.5$ and maximum consumption rate $\bar{x}\left(\bar{y}_{1}+\bar{y}_{2}\right)=0.25$.

\subsubsection{A positive effect of incongruity in social relevance}

The effect of incongruity or asymmetry ${ }^{5}$ in the social relevances on the game tragicness can also be observed in Figure 3(a). Moving away from the line of zero asymmetry (the 45-degree line through the origin) horizontally in either direction results in a lower tragicness level. This effect of asymmetry on the tragicness is less pronounced for small deviations in asymmetry, which can be seen from the decreased slope of the level curves when they are near the line of zero asymmetry. When the asymmetry is large, this effect is more significant, which can be seen from the sudden slanting of the level curves when further away from zero asymmetry. Thus strong incongruity or heterogeneity in social relevance corresponds to less tragic games.

Incongruity has a similar effect on the resource stock and total consumption rates, which can be seen in Figure 3(b) and Figure 3(c) respectively. In general, an increase in incongruity increases both. Note that the discontinuity of the level curves, which was present in Figure 3(a) is also present here at exactly the same points in the

\footnotetext{
${ }^{5}$ Henceforth, we use the terms "asymmetry" and "incongruity" interchangeably with "heterogeneity", a term commonly associated with large populations, in contrast to the system represented by (3), which considers two consumer groups only.
} 
parameter space. However the behaviour of the curves is different in the centre. They are now concave, which means that small deviations in asymmetry are detrimental to the resource stock and the consumed quantity. However, large deviations in asymmetry are beneficial.

\subsubsection{Higher social relevance increases individual consumption}

In general, higher social relevance increases individual group consumption. Figure 3(d) depicts how individual consumption of a group changes as the social relevances are varied. The level curves in Figure 3(d) show three different behaviours. Near the symmetric line where $v_{1}=v_{2}$, the consumption of the focal group increases with respect to the social relevance of both groups. However, this increase is more pronounced with respect to changes in its own social relevance, than that of the other group. Variation in the other group's social relevance does not affect the group's consumption, when the social relevance of the other group is low. Conversely, for low values of its own social relevance, the group's consumption is affected only by changes in the social relevance of the other group.

Also note that a group's individual consumption increases monotonically with the other group's social relevance only when its own social relevance is high. When this is not the case, a mode is encountered in the region where the other group's social relevance is high enough - see the plateau in the upper right area of Figure 3(d).

Furthermore, a group with a higher social relevance consumes more, than a group with a lower social relevance. Figure 3(e) depicts the difference in consumption $\bar{x}\left(\bar{y}_{1}-\bar{y}_{2}\right)$ which always stays positive to the right of the symmetric line $\left(v_{1}=v_{2}\right)$ and always negative to the left of this line. Difference in consumption is exactly zero when 
social relevance for both consumer groups are the same.

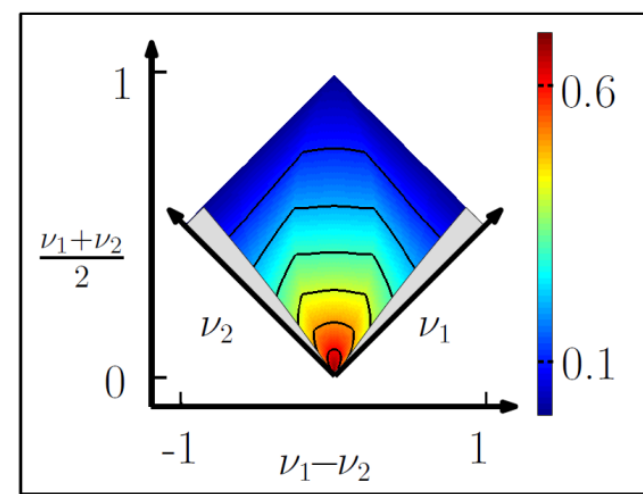

(a): Game tragicness

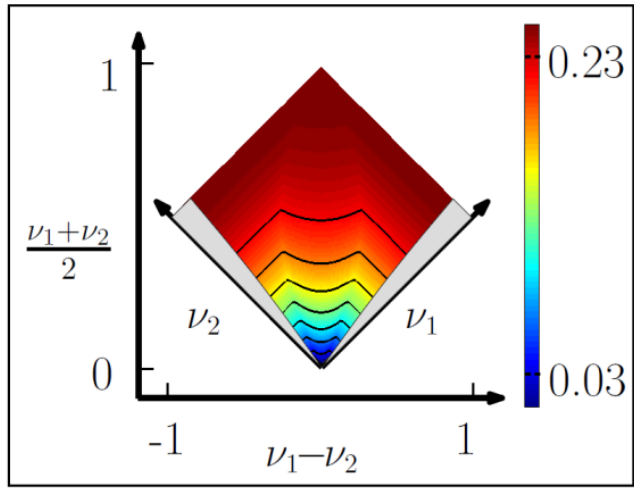

(c): Total consumption $\bar{x}\left(\bar{y}_{1}+\bar{y}_{2}\right)$

Figure 3: Plots for different characteristics of the consumption game at Nash equilibrium, shown as the difference and average level of the social relevances $v_{1}, v_{2}$ are varied. For completeness the $v_{1}$ and $v_{2}$ axes have also been shown in each plot. The grey areas mark regions for which stability is not guaranteed. The plots for both groups are symmetric to each other, and so the conclusions drawn here for group 1 also apply to group 2 .

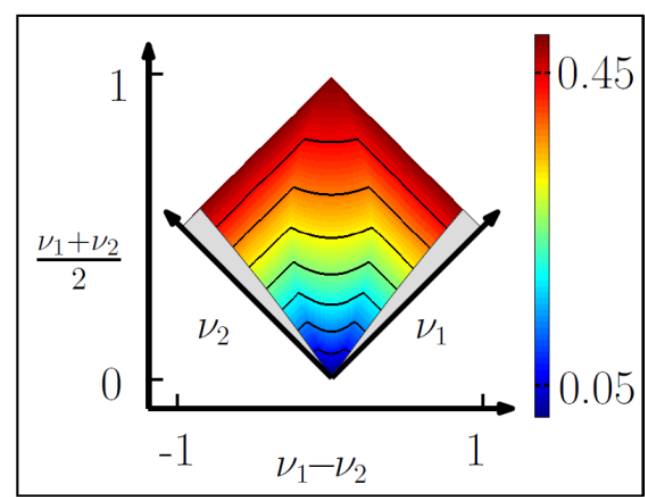

(b): Resource quantity $\bar{x}$

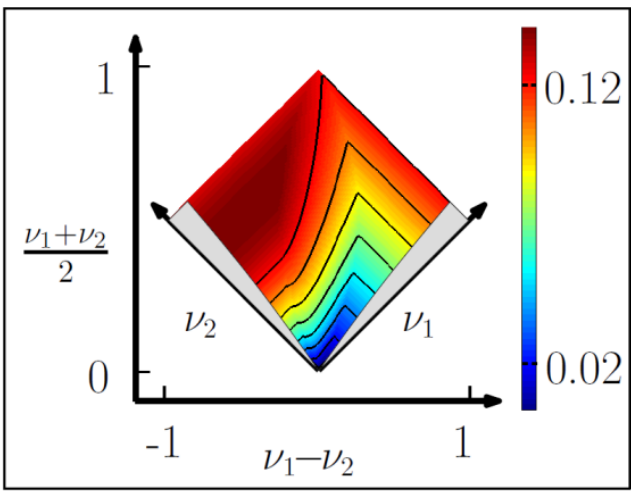

(d): Individual consumption $\bar{x} \bar{y}_{1}$

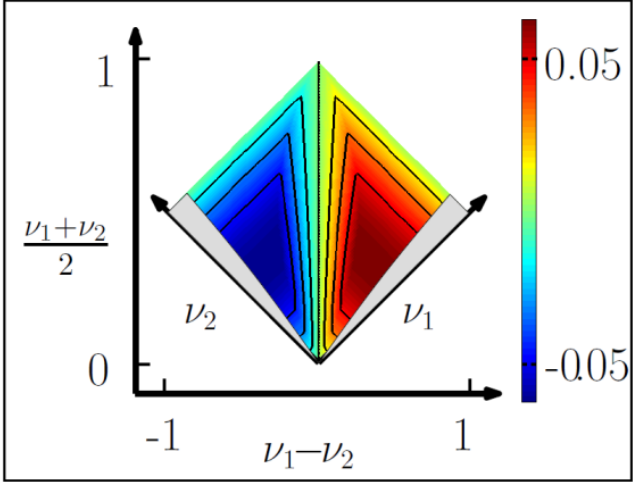

(e): Consumption equity $\bar{x}\left(\bar{y}_{1}-\bar{y}_{2}\right)$ 


\subsubsection{Equity in social relevance induces equity in consumption}

Figure $3(\mathrm{e})$ shows the behaviour of the equity measure $\bar{x}\left(\bar{y}_{1}-\bar{y}_{2}\right)$ as a function of the average of the social relevances and their asymmetry. When the social relevances are close to equal (near the centre of the plot), the isolines are nearly vertical which shows that the average quantity of the social relevances does not affect equity in consumption. However, the equity is affected significantly by the asymmetry in social relevances. This can be traced by starting in the middle of the plot and observing the difference in consumption as we move horizontally in either direction. The difference in consumption is increased as we increase asymmetry until a peak is reached after which it decreases slightly. This shows that equity in consumption is the highest, when the social relevances are close to each other in value.

\section{Discussion}

Leon Festinger's theory on Social Comparison Processes (Festinger 1954) provides the rationale for Social-Ecological Relevance (SER), which is the core theoretical concept of the model in (Mosler \& Brucks 2003). SER is one-dimensional and so the preference given to social factors is the complement of that given to ecological factors, which represents the bipolarity between social and physical dimensions. The model that we put forward here also captures this concept as $\alpha_{i}$ and $v_{i}$ can be interpreted as the relative preferences given to the ecological and social factors respectively and as they are the complements of each other they depict the bipolarity inherent in the underlying social comparison process.

Opinions on the effect of heterogeneity on successful resource management are highly variable (Varughese \& Ostrom 2001; Bardhan \& Dayton-Johnson 2002; Chand, N. Kerr \& Bigsby 2015) and range from negative to positive to no effect at all. Our 
model presents mixed results regarding heterogeneity in general, which suggests that heterogeneity is important in different ways across different factors. Section 3.2 shows that incongruity in environmental concerns promotes free-riding, whereas Section 3.3 shows that incongruity in social relevance helps avoid tragedies. Note that they are expressed via the parameters $\rho_{i}$ and $v_{i}$, respectively, which are different in nature. Hence, while our model does not provide a straight-forward verdict on the role of heterogeneity, it does contribute to the debate by indicating, that heterogeneity may need to be exposed to a classification, which is deeper than the conventional classification of economic, non-economic and socio-cultural heterogeneity to correctly establish its correlation with successful resource management.

Despite the aforementioned strengths of the model, there also exist some limitations. First, the psychological parameters of the system have been assumed to be constant over time. Although in some cases a society may exhibit unvarying characteristics (Friedkin 2006) for short enough time span, it is understood that in general, societies in the real-world are in a constant state of change. Second, the analysis presented here has been conducted for two consuming entities. Although the extension to an arbitrary number of consumers is fairly straightforward, the analysis of Section 3 does not scale very easily to higher dimensions and must be conducted in some other form. Third, the assumption of perfect access of each consumer group, to information on the consumption of the other groups, is obviously restrictive of a true representation of reality. Future work can be directed towards relaxing this assumption, and observing the implications it may have, on the conclusions drawn from the model we put forward here. Fourth, the conclusions of the current study have all been drawn at the steady state, while ignoring the transient behaviour. While a study of the steady state has its own importance, transient analyses might be able to provide additional insights 
for several resource exploitation problems (Perman 2003). It is however beyond the scope of this paper and an obvious direction for future research.

The basic model of (3) can be extended to an arbitrary number of consumer groups, which would then make it possible to incorporate the underlying social network (consumers are affected only by the individuals they are socially connected to). This inclusion of the underlying social network in the model makes it possible to look at the characteristics of socio-ecological systems with large consuming populations, as a function of network topology ( (Easley \& Kleinberg 2010) gives an excellent introduction to the application of network science to socio-economic systems). It would also enable us to examine conditions on the network for which we are able to combine the individual consumers and view the system in terms of aggregate consumption of different consumer groups. In fact, the dynamics of the society may also be viewed as a single entity by modelling it as a single consumer group with certain conditions of homogeneity holding within the network. Needless to say, the extensions to the basic model are many and there exist a number of directions for future research.

\section{Acknowledgements}

Part of the research was developed in the Young Scientists Summer Program at the International Institute for Systems Analysis, Laxenburg (Austria). The first author received a grant for participation under an IIASA-Pakistan cooperation by the Government of Pakistan. The authors would also like to acknowledge Adam French and Tariq Samad for their valuable feedback on earlier versions of the manuscript.

\section{Literature cited}

Anderies, JM 2000, 'On modeling human behavior and institutions in simple ecological economic systems', Ecological Economics, vol 35, no. 3, pp. 393-412. 
Anderson, JM 1974, 'A Model for"The Tragedy of the Commons"', Systems, Man and Cybernetics, IEEE Transactions on, vol 4, no. 1, pp. 103-105.

Anshelevich, E, Dasgupta, A, Kleinberg, J, Tardos, E, Wexler, T \& Roughgarden, T 2008, 'The price of stability for network design with fair cost allocation', SIAM Journal on Computing, vol 38, no. 4, pp. 1602-1623.

Bardhan, P \& Dayton-Johnson, J 2002, 'Unequal irrigators: heterogeneity and commons management in large-scale multivariate research', in The drama of the commons.

Birch, CP 1999, 'A new generalized logistic sigmoid growth equation compared with the Richards growth equation', Annals of Botany, pp. 713-723.

Bousquet, F \& Le Page, C 2004, 'Multi-agent simulations and ecosystem management: a review', ecollogical modelling, vol 176, no. 3, pp. 313-332.

Bray, DB, Merino-Pérez, L, Negreros-Castillo, P, Segura-Warnholtz, G, Torres-Rojo, JM \& Vester, HF 2003, 'Mexico's community-managed forests as a global model for sustainable landscapes', Conservation Biology.

Brucks, WM \& Mosler, HJ 2011, 'Information preferences and corresponding consumption behavior in common pool resource management', Social Psychology, vol 42, no. 4, pp. 261-270.

Chakravarty, SR 2015, 'Social Polarization', in Inequality, Polarization and Conflict, Springer India.

Chand, N, N. Kerr, G \& Bigsby, H 2015, 'Production efficiency of community forest management in Nepal', Forest Policy and Economics, vol 50, pp. 172-179.

Chazdon, RL 2003, 'Tropical forest recovery: legacies of human impact and natural disturbances', Perspectives in Plant Ecology, evolution and systematics.

Cole, DH \& Grossman, PZ 2010, 'Institutions matter! Why the Herder Problem is not a Prisoner's Dilemma', Theory and Decision, vol 69, no. 2, pp. 219-231. 
De Groot, JI \& Steg, L 2010, 'Relationships between value orientations, self-determined motivational types and pro-environmental behavioural intentions', Journal of Environmental Psychology, vol 30, no. 4, pp. 368-378.

Deadman, PJ 1999, 'Modelling individual behaviour and group performance in an intelligent agent-based simulation of the tragedy of the commons', Journal of Environmental Management, vol 56, no. 3, pp. 159-172.

Diekert, FK 2012, 'The Tragedy of the Commons from a Game-Theoretic Perspective', Sustainability, vol 4, no. 8, pp. 1776-1786.

Doebeli, M \& Hauert, C 2005, 'Models of cooperation based on the Prisoner's Dilemma and the Snowdrift game', Ecology Letters, vol 8, no. 7, pp. 748-7668.

Easley, D \& Kleinberg, J 2010, Networks, crowds, and markets: Reasoning about a highly connected world, Cambridge University Press.

Fekedulegn, D, Mac Siúrtáin, MP \& Colbert, JJ 1999, 'Parameter Estimation of Nonlinear Models in Forestry', Silva Fennica, pp. 327-336.

Festinger, L 1954, 'A theory of social comparison processes', Human relations, vol 7 , no. 2, pp. 117-140.

Feuillette, S, Bousquet, F \& Le Goulven, P 2003, 'SINUSE: a multi-agent model to negotiate water demand management on a free access water table', Environmental Modelling \& Software, vol 18, no. 5, pp. 413-427.

Franklin, GF, Powell, JD \& Emami-Naeini, A 1994, Feedback control of dynamic systems, Addison-Wesley, Reading, MA.

Friedkin, NE 2006, A structural theory of social influence, Cambridge University Press. Gärling, T, Fujii, S, Gärling, A \& Jakobsson, C 2003, 'moderating effects of social value orientation on determinants of pro-environmental behaviour intention', Journal of environmental psychology, vol 23, pp. 1-9. 
Gordon, HS 1954, 'The economic theory of a common-property resource: the fishery', Bulletin of Mathematical Biology, vol 53, no. 1, pp. 231-252.

Hardin, G 1968, 'The tragedy of the commons', science, vol 162, no. 3859, pp. 12431248.

Jager, W, Janssen, MA, De Vries, HJM, De Greef, J \& Vlek, CAJ 2000, 'Behaviour in commons dilemmas: Homo economicus and Homo psychologicus in an ecologicaleconomic model', Ecological economics, vol 35, no. 3, pp. 357-379.

Jager, W \& Mosler, HJ 2007, 'Simulating human behavior for understanding and managing environmental resource use', Journal of Social Issues, vol 63, no. 1, pp. 97 116.

Kerschner, C 2010, 'Economic de-growth vs. steady-state economy', Journal of Cleaner Production, vol 18, no. 6, pp. 544-551.

Khalil, HK 1996, Nonlinear systems, Prentice hall, New Jersey.

Koutsoupias, E \& Papadimitriou, C 2009, 'Worst-case equilibria', Computer science review, vol 3, no. 2, pp. 65-69.

Madani, K 2010, 'Game theory and water resources', Journal of Hydrology, vol 381, no. 3, pp. 225-238.

Meadows, DH, Meadows, DL, Randers, J \& Behrens, WW 1972, The limits to growth, New York.

Mosler, H-J \& Brucks, WM 2003, 'Integrating commons dilemma findings in a general dynamic model of cooperative behavior in resource crises', European Journal of Social Psychology, vol 33, no. 1, pp. 119-133.

Ostrom, E 1990, Governing the commons: The evolution of institutions for collective action, Cambridge University Press. 
Ostrom, E, Gardner, R \& Walker, J 1994, Rules, games, and common-pool resources, University of Michigan Press.

Parrachino, I, Dinar, A \& Patrone, F 2006, 'Cooperative game theory and its application to natural, environmental, and water resource issues', World Bank Policy Research Working Paper, 4074.

Perman, R 2003, Natural resource and environmental economics, Pearson Education. Roopnarine, P 2013, 'Ecology and the Tragedy of the Commons', sustainability, vol 5, no. 2 , pp. $749-773$.

Rutte, CG, Wilke, HA \& Messick, DM 1987, 'Scarcity or abundance caused by people or the environment as determinants of behavior in the resource dilemma', Journal of Experimental Social Psychology, vol 23, no. 3, pp. 208-216.

Samuelson, CD, Messick, DM, Rutte, C \& Wilke, H 1984, 'Individual and structural solutions to resource dilemmas in two cultures', Journal of Personality and Social Psychology, vol 47, no. 1, p. 94.

Simon, HA 1976, Administrative Behavior: A Study of Decision Making Processes in Administrative Organization, Harper, New York.

Torabi, B, Saadatkhah, H \& Soltani, A 2014, 'Evaluating Mechanistic Models in Growth Analysis of Safflower', Agriculture Science Developments.

Van Lange, PA, Joireman, J, Parks, CD \& Van Dijk, E 2013, 'The psychology of social dilemmas: A review', Organizational Behavior and Human Decision Processes, vol 120, no. 2, pp. 125-141.

Van Liere, KD \& Dunlap, RE 1980, 'The social bases of environmental concern: A review of hypotheses, explanations and empirical evidence', public opinion quarterly, vol 44, no. 2, pp. 181-197. 
Varughese, G \& Ostrom, E 2001, 'The contested role of heterogeneity in collective action: some evidence from community forestry in Nepal', World development, vol 29, no. 5 , pp. 747-765.

Werker, AR \& Jaggard, KW 1997, 'Modelling asymmetrical growth curves that rise and then fall: applications to foliage dynamics of sugar beet (Beta vulgaris L.)', Annals of Botany.

Worm, B,HR,BJK, Branch, TA, Collie, JS, Costello, C, Fogarty, MJ, Fulton, EA, Hutchings, JA, Jennings, S \& Jensen, OP 2009, 'Rebuilding global fisheries', Science. Zwiers, M, Kleinhans, R \& Van Ham, M 2015, 'Divided Cities: Increasing SocioSpatial Polarization within Large Cities in the Netherlands', IZA Discussion Papers, 8882.

\section{Appendix}

\subsection{Stability}

Here we investigate the stability of the equilibrium of system (3). Before proceeding with the analysis, let us point out that there are two degenerate cases of the equilibrium (4). First, if both groups have extremely low social value, i.e., if $v_{1}=v_{2}=0$, then an infinite number of equilibria exist if the groups are equally environmental $\left(\rho_{1}=\rho_{2}\right)$, otherwise no equilibrium exists. Second, if both groups have extremely high social value, i.e., if $v_{1}=v_{2}=1$, then infinite equilibria exist for all values of $\rho_{1}$ and $\rho_{2}$. We ignore these pathological cases in further analyses.

The stability of the equilibrium point of system (3) can be investigated by examining the eigenvalues of the linearized system around that point. The Jacobian matrix of (3) is given by 


$$
\left[\begin{array}{ccc}
1-2 x-y_{1}-y_{2} & -x & -x \\
b_{1} \alpha_{1} & -b_{1} v_{1} & b_{1} v_{1} \\
b_{2} \alpha_{2} & b_{2} v_{2} & -b_{2} v_{2}
\end{array}\right]
$$

Evaluation at the equilibrium $\left(\bar{x}, \bar{y}_{1}, \bar{y}_{2}\right)$ results in

$$
\left[\begin{array}{ccc}
-\frac{\alpha_{1} v_{2} \rho_{1}+\alpha_{2} v_{1} \rho_{2}}{\alpha_{2} v_{1}+\alpha_{1} v_{2}} & -\frac{\alpha_{1} v_{2} \rho_{1}+\alpha_{2} v_{1} \rho_{2}}{\alpha_{2} v_{1}+\alpha_{1} v_{2}} & -\frac{\alpha_{1} v_{2} \rho_{1}+\alpha_{2} v_{1} \rho_{2}}{\alpha_{2} v_{1}+\alpha_{1} v_{2}} \\
b_{1} \alpha_{1} & -b_{1} v_{1} & b_{1} v_{1} \\
b_{2} \alpha_{2} & b_{2} v_{2} & -b_{2} v_{2}
\end{array}\right]
$$

The eigenvalues of this matrix are given by the roots of the following characteristic polynomial

$$
\begin{aligned}
& p(\lambda)=2 b_{1} b_{2}\left(\alpha_{2} v_{1} \rho_{2}+\alpha_{1} v_{2} \rho_{1}\right)+\frac{\alpha_{2} v_{1} \rho_{2}+\alpha_{1} v_{2} \rho_{1}}{\alpha_{2} v_{1}+\alpha_{1} v_{2}}\left(b_{1}+b_{2}\right) \lambda \\
& +\frac{1}{\alpha_{2} v_{1}+\alpha_{1} v_{2}}\left(\left(b_{1} v_{1}+b_{2} v_{2}\right)\left(\alpha_{2} v_{1}+\alpha_{1} v_{2}\right)+\alpha_{2} v_{1} \rho_{2}+\alpha_{1} v_{2} \rho_{1}\right) \lambda^{2}+\lambda^{3} .
\end{aligned}
$$

The final expressions for the roots of this polynomial are not simple enough to work with analytically. It can be noted, however, that all the coefficients of $p(\lambda)$ are positive, which is a necessary (but not sufficient) condition for all roots to be negative and, hence, the linearized system to be stable. Whether or not the equilibrium is indeed stable can be checked by Routh's Stability Criterion (Franklin, Powell \& Emami-Naeini 1994), which for cubic polynomials of the form $a x^{3}+b x^{2}+c x+d$ is given by $b c>$ $a d$. For $p(\lambda)$, this inequality is given as

$$
\begin{gathered}
\frac{\alpha_{2} v_{1} \rho_{2}+\alpha_{1} v_{2} \rho_{1}}{\left(\alpha_{2} v_{1}+\alpha_{1} v_{2}\right)^{2}}\left(b_{1}+b_{2}\right)\left(\left(b_{1} v_{1}+b_{2} v_{2}\right)\left(\alpha_{2} v_{1}+\alpha_{1} v_{2}\right)+\alpha_{2} v_{1} \rho_{2}+\alpha_{1} v_{2} \rho_{1}\right) \\
>2 b_{1} b_{2}\left(\alpha_{2} v_{1} \rho_{2}+\alpha_{1} v_{2} \rho_{1}\right)
\end{gathered}
$$

which is simplified to

$$
\frac{\left(b_{1}+b_{2}\right)}{\left(\alpha_{2} v_{1}+\alpha_{1} v_{2}\right)^{2}}\left(\begin{array}{c}
\left(b_{1} v_{1}+b_{2} v_{2}\right)\left(\alpha_{2} v_{1}+\alpha_{1} v_{2}\right) \\
+\alpha_{2} v_{1} \rho_{2}+\alpha_{1} v_{2} \rho_{1}
\end{array}\right)-2 b_{1} b_{2}>0
$$


It can be checked that there exist $b_{1}, b_{2}, \rho_{1}, \rho_{2}, v_{1}, v_{2}$, for which (5) does not hold $\left(b_{1}=0.2, b_{2}=0.1, \rho_{1}=0.001, \rho_{2}=0.1, v_{1}=0.01, v_{2}=0.9\right.$ is an example).

On the other hand, the following inequality

$$
\frac{\left(b_{1}+b_{2}\right)\left(b_{1} v_{1}+b_{2} v_{2}\right)}{\left(\alpha_{2} v_{1}+\alpha_{1} v_{2}\right)^{2}}-2 b_{1} b_{2}>0 \text {, }
$$

clearly offers a sufficient condition for (5) to hold. It can be simplified to

$$
q(b)=v_{1} b^{2}+\left(v_{1}\left(2 v_{2}-1\right)+v_{2}\left(2 v_{1}-1\right)\right) b+v_{2}>0
$$

where $b=b_{1} / b_{2}$. Note that the right hand side of (6) is a quadratic polynomial in $b>$ 0 . As $v_{i}>0$, the graph of $q(\cdot)$ points upwards and the roots are either complex conjugates or both real and positive. In what follows we examine the implications of each case in detail

Case 1: $q(\cdot)$ has complex conjugate roots i.e., the discriminant is negative.

$$
\left(v_{1}\left(2 v_{2}-1\right)+v_{2}\left(2 v_{1}-1\right)\right)^{2}-4 v_{1} v_{2}<0
$$

In this case (6) will always hold as the graph of the quadratic polynomial will lie above the horizontal axis.

Case 2: $q(\cdot)$ has both roots real and positive. This happens if

$$
\left(v_{1}\left(2 v_{2}-1\right)+v_{2}\left(2 v_{1}-1\right)\right)^{2}-4 v_{1} v_{2} \geq 0 \text {, }
$$

and

$$
-v_{1}\left(2 v_{2}-1\right)-v_{2}\left(2 v_{1}-1\right)>0 \text {. }
$$

In this case (6) does not hold for those values of $b$ which lie between the roots of $q(\cdot)$ 


\section{complex conjugate $\square$ real and positive}

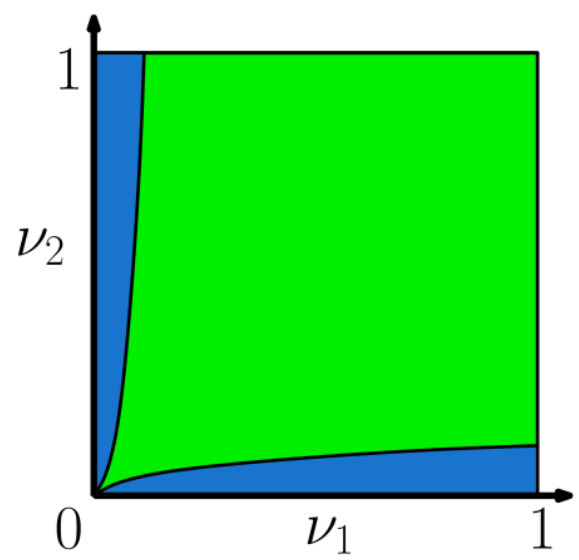

Figure 4: Evaluation of the roots of $q(\cdot)$.

Thus (7) is a sufficient condition for stability. However, if (7) does not hold, then the system is stable, if (8)-(9) hold true and $b$ does not lie between the roots of $q(\cdot)$. The regions in the parameter space where these inequalities hold true are shown in Figure 4.

\section{stable $\square$ unstable}
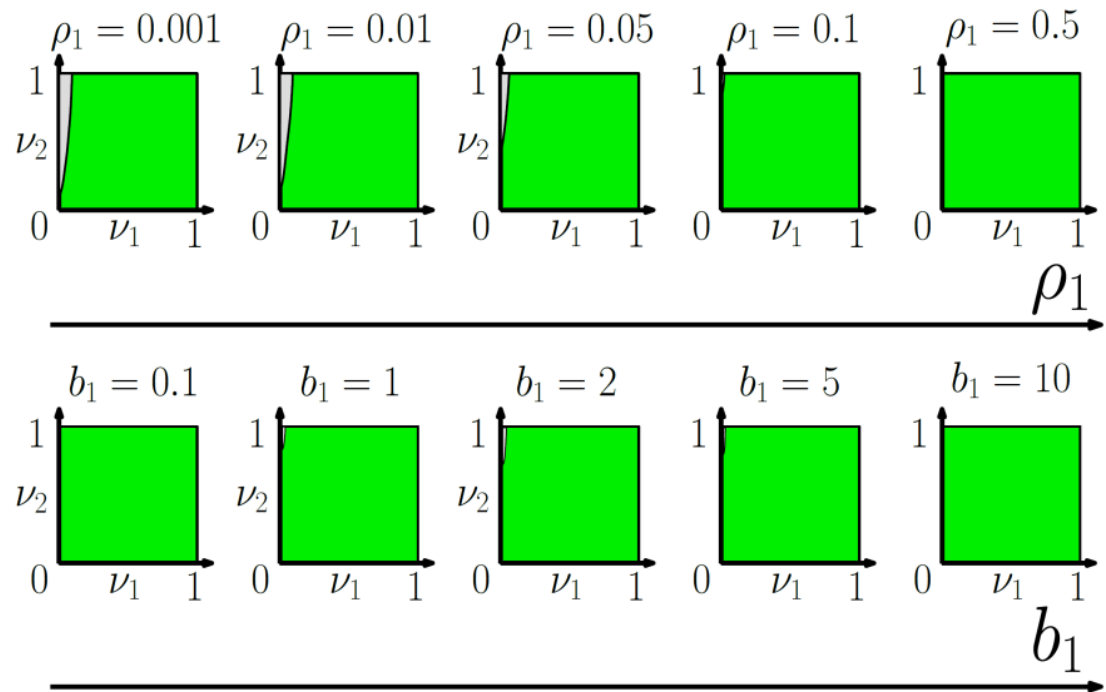

Figure 5: Here we evaluate the stability of (3), through inequality (5), as the parameters are varied. The nominal values for the parameters are $b_{1}=0.2, b_{2}=$ 0.1 , and $\rho_{1}=\rho_{2}=0.1$. The plots obtained by interchanging the indices of the groups are symmetric to the ones displayed above and thus are not shown here. 
Figure 5 shows an exhaustive simulation in the space $v_{1}, v_{2} \in(0,1)$ to determine when condition (5) holds. We see that stability is guaranteed in a major portion of the $\left(v_{1}, v_{2}\right)$ space regardless of the values of the rest of the parameters. Also note that for small values of $r$ and $\left(\rho_{1}, \rho_{2}\right),(5)$ simply reduces to (6).

\subsubsection{Global Stability}

Above, we derived conditions on the parameters for which the equilibrium is locally stable. Local stability of the equilibrium implies that there exists at least some region of the phase space, such that if the system is initiated from any point in this region the dynamics asymptotically approach the equilibrium. This region exists as a non-empty neighbourhood of the equilibrium, whose size may be determined through a global stability analysis, thus complementing the local stability analysis. However in this study, we do not focus on the transient dynamics (which would be explicitly dependent on the initial conditions), but rather on the steady state. Moreover, since a locally stable equilibrium point is reachable from at least some sub-set of the phase space, we argue that establishing local stability alone is sufficient in order to justify the conclusions drawn in this study. Nonetheless, the insights gained from a global stability analysis of the equilibrium can help us gain additional understanding of the model.

In what follows, we summarize the stability properties of the system through a numerical simulation of probabilistic nature. It is important to note that there exist rigorous analytical techniques (Khalil 1996), that yield sufficient (but not necessary) conditions for global stability for non-linear systems. However, due to the deceptively complex nature of our system (including its dimension) and limitations on the scope of this study, the application of these techniques to our model has been designated to a future study. For the sake of this study however, the results of the simulation described 
below suggest that for physically realizable values of the parameters and initial conditions, there do not exist any periodic or chaotic solutions, and thus all of the considered region of the phase space lies within the region of attraction for the equilibrium.

The simulation consists of the following steps. First a parameter set $\left(\rho_{1}, \rho_{2}, v_{1}, v_{2}, b_{1}, b_{2}\right)$ is generated at random. Here $\rho_{i}, v_{i} \in(0,1)$ and $b_{i} \in\left(0, b_{\max }\right)$ where $b_{\max }$ is to be chosen independently. The parameter sets generated must lie in the region where the system is locally stable. For the generated parameter set, 100 trajectories are initiated at random. The initial points $\left(x(0), y_{1}(0), y_{2}(0)\right)$ are generated such that $x(0) \in\left(0, x_{\max }\right)$ and $y_{i}(0) \in\left(y_{\min }, y_{\max }\right)$, where $x_{\max }, y_{\min }, y_{\max }$ are also chosen independently. The trajectories are then run for time $\tau_{\text {final }}=5000$. With time step of 250 units, we register the percentage of total trajectories (for that particular parameter set) for which the norm of the derivative $\left\|\dot{Z}\left(\tau_{k}\right)\right\|$ (where $Z\left(\tau_{k}\right)=$ $\left.\left[x\left(\tau_{k}\right), y_{1}\left(\tau_{k}\right), y_{2}\left(\tau_{k}\right)\right]\left(\tau_{k}=0,250,500, \ldots, 5000\right)\right)$ is less than some small value $\epsilon$, and plot it against time. The process is then repeated for 100 parameter sets. Thus there are a 100 points corresponding to each value of $\tau_{k}$.

The limits for the random parameters and variables are chosen so that the generated values fall within a range that mimics real-world behavior. This has been done as follows

1. If the aggregate consumption effort is negative, then the resource can grow over the natural carrying capacity, i.e., $x(\tau)$ may take on values greater than one, which in the context of the model means that the resource grows beyond the carrying capacity. However even then there is a limit to how much beyond the natural carrying capacity the resource quantity can be stretched. In the case of forests, for example, using fertilizers and planting 
trees can act as negative extraction efforts leading to the forest biomass growing over the natural carrying capacity. However, the limited amount of light coming onto the Earth, competition for light between trees and simply the space constraint will not allow the biomass to grow unboundedly and thus there will be some limit to it; similar examples can be envisioned for other resources as well. In this simulation, we choose the limit $x_{\max }$ to be 10 units of natural carrying capacity.

2. The value of $x_{\max }$ is used to determine $y_{\min }$ as follows. Let $y$ be a constant total consumption effort, i.e., $\dot{x}(\tau)=x(\tau)(1-x(\tau))-y x(\tau)$. Solving for $x(\tau)$ analytically it can be seen that as $\tau \rightarrow \infty, x(\tau) \rightarrow 1-y$ for all $x(0) \in$ $\mathbb{R}$ and all $y<1$. This means that the lower limit for $1-y$ should be near the upper limit for $x(\tau)$, i.e., $x_{\max }$, and so we choose $y_{\min }=-10$.

3. We determine $y_{\max }$ as follows. Again, assuming $y$ to be a constant, but positive consumption effort, we seek to find a value for $y$ (to be regarded as $\left.y_{\max }\right)$ such that the corresponding solution $x\left(\cdot \mid y_{\max }\right)$ approximates the solution $x(\cdot \mid \infty)$. Since $x(\tau \mid \infty) \equiv 0$ the norm $\|x(\tau \mid y)\|_{L 2}=\sqrt{\int_{0}^{\infty} x^{2}(\tau) d \tau}$, will be small enough for all $x(0) \in[0,1]$. We choose $y=550$ which results in $\|x(\tau)\|_{L 2}=0.02$. Since $y$ is interpreted as the total consumption and we have two consumer groups in the model, we set $y_{\max }=225$.

4. To determine $b_{\max }$ we note that $b_{i}$ is interpreted as the susceptibility of group $i$ to change in its consumption. Such a parameter is also found in similar models of opinion formation, where it is estimated to lie between zero and one (Friedkin 2006). Here we take the upper limit as $b_{\max }=10$ allowing some margin. 


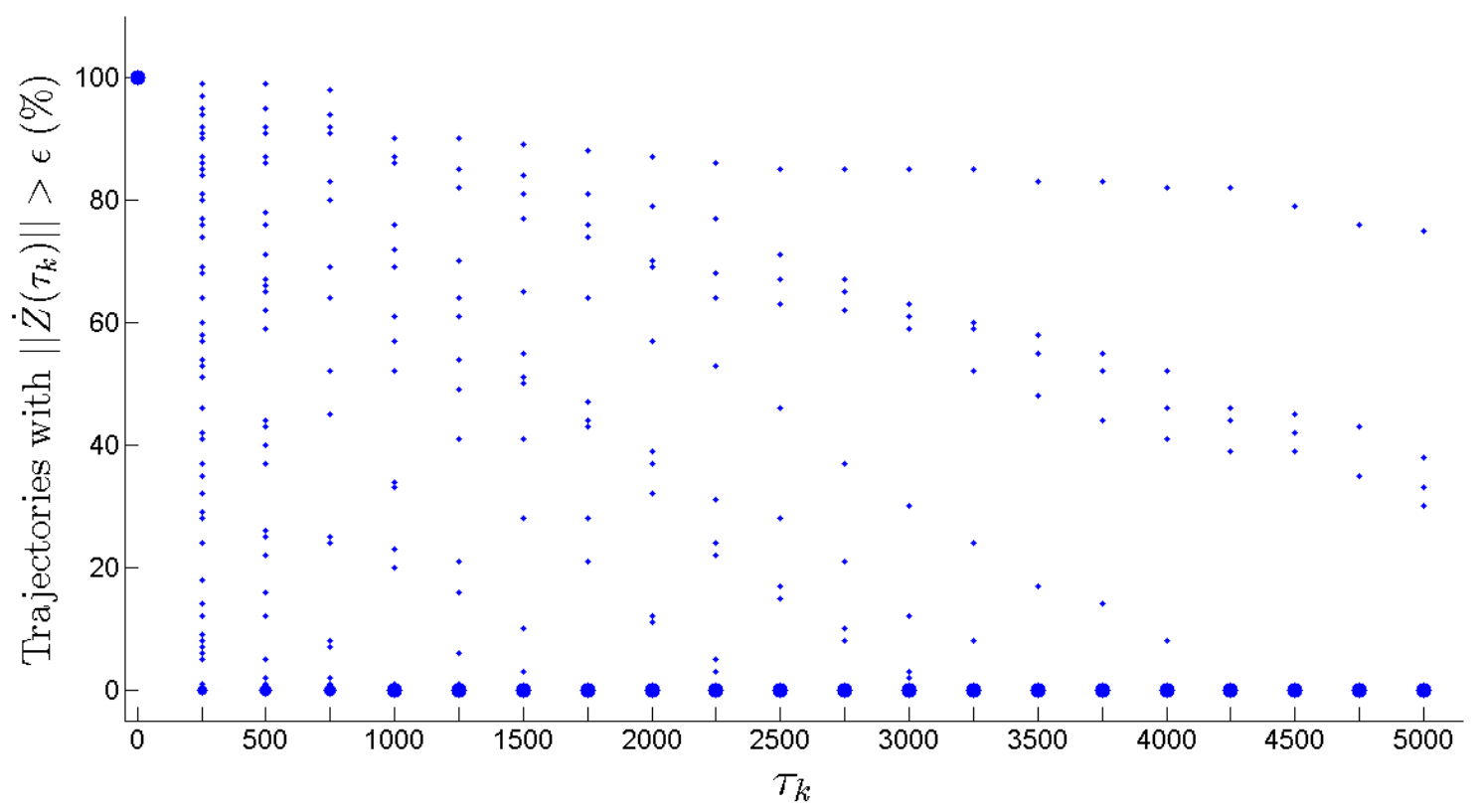

Figure 6: The percentage of trajectories for which the derivative is sufficiently small, plotted as time progresses, for each parameter set. For this simulation $b_{\max }=$ $10, \mathrm{x}_{\max }=10, \mathrm{y}_{\min }=-10, \mathrm{y}_{\max }=225$ and $\epsilon=0.01$.

Figure 6 shows the resulting plot. Each point corresponds to an individual simulation with the generated parameter set and initial conditions; $100 * 100=10,000$ simulations were carried out (of which we resampled $14 \%$ of the cases when computational errors were detected - the source of these errors was the failure of the ODE solver to perform the integration; for this simulation, an adaptive version of the 4th order Runge-Kutta method was used). The plot suggests that within the considered time horizon of 5,000 dimensionless time units, most of the simulated trajectories reach the origin with the given small precision; those which have not reached the origin yet, follow a decreasing trend which gives some confidence that they will reach it over longer time horizons. So at least for the cases that are randomly generated, the equilibrium seems to be asymptotically stable.

\subsection{Nash equilibrium}

Here we formally introduce the resource consumption game. Each player $i=1,2$ 
chooses her level of environmentalism $\rho_{i}$ and her social relevance $v_{i}$ (note that $\alpha_{i}=$ $1-v_{i}$ and so $\alpha_{i}$ is determined by the choice of $v_{i}$ ). Thus the strategy set for each individual $i$ is given as $\tilde{S}_{i}=\left\{\rho_{i}, v_{i}\right\}$. The payoff $\tilde{\pi}_{i}$ that each individual receives is equal to the amount of resource $\bar{x} \bar{y}_{i}$ that individual harvests at steady state, where $\left(\bar{x}, \bar{y}_{1}, \bar{y}_{2}\right)$ are given by (4). Note that $b_{i}$ does not affect the equilibrium and thus is not included in the strategy set $\tilde{S}_{i}$. The game is defined as a 3-tuple $\tilde{G}=\left\langle I,\left(\tilde{S}_{i}\right),\left(\tilde{\pi}_{i}\right)\right\rangle$ where $I=\{1,2\}$ denotes the set of players, $\tilde{S}_{i}=[0,1] \times[01] ; i \in I$ is the strategy space and $\tilde{\pi}_{i}: \tilde{S}_{i} \rightarrow$ $\mathbb{R} ; i \in I$ is the payoff function for consumer $i$.

For the two-player game $\tilde{G}$, the pay-off functions are given as $\tilde{\pi}_{1}\left(\rho_{1}, v_{1}, \rho_{2}, v_{2}\right)=\bar{x} \bar{y}_{1}$ and $\tilde{\pi}_{2}\left(\rho_{1}, v_{1}, \rho_{2}, v_{2}\right)=\bar{x} \bar{y}_{2}$, where

$$
\begin{aligned}
& \bar{x}\left(\rho_{1}, v_{1}, \rho_{2}, v_{2}\right)=\frac{\alpha_{2} \rho_{2} v_{1}+\alpha_{1} \rho_{1} v_{2}}{\alpha_{2} v_{1}+\alpha_{1} v_{2}} \\
& \bar{y}_{1}\left(\rho_{1}, v_{1}, \rho_{2}, v_{2}\right)=\frac{\left(1-\rho_{2}\right) \alpha_{2} v_{1}+\left(1-\rho_{1}\right) \alpha_{1} v_{2}-\left(\rho_{1}-\rho_{2}\right) \alpha_{1} \alpha_{2}}{2\left(\alpha_{2} v_{1}+\alpha_{1} v_{2}\right)} \\
& \bar{y}_{2}\left(\rho_{1}, v_{1}, \rho_{2}, v_{2}\right)=\frac{\left(1-\rho_{2}\right) \alpha_{2} v_{1}+\left(1-\rho_{1}\right) \alpha_{1} v_{2}-\left(\rho_{2}-\rho_{1}\right) \alpha_{1} \alpha_{2}}{2\left(\alpha_{2} v_{1}+\alpha_{1} v_{2}\right)}
\end{aligned}
$$

The best response of player $i$ is the strategy $\left(\tilde{\rho}_{i}, \tilde{v}_{i}\right)$ that maximizes $\tilde{\pi}_{i}$ for a fixed strategy of the other player $j \neq i$. The best response functions are thus given as

$$
\left(\tilde{\rho}_{i}, \tilde{v}_{i}\right)=\arg \max _{\rho_{j}, v_{j}} \tilde{\pi}_{i}\left(\rho_{i}, v_{i}, \rho_{j}, v_{j}\right)
$$

By calculating the partial derivatives and putting them to zero, we find that for a fixed pair $\left(\rho_{j}, v_{j}\right), \tilde{\pi}_{i}\left(\because, \rho_{j}, v_{j}\right)$ is maximized not at a single point but along the following curve

$$
\tilde{\rho}_{i}=\left(\frac{\left(1-v_{j}\right)\left(\rho_{j}-v_{j}\left(1-\rho_{j}\right)\right)}{2 v_{j}}\right) \frac{1}{\tilde{v}_{i}-1}+\frac{\rho_{j}+v_{j}\left(1-\rho_{j}\right)\left(2 v_{j}-1\right)}{2 v_{j}} ; i, j \in I ; i \neq j .
$$

A realization of this curve is shown in Figure 7. 


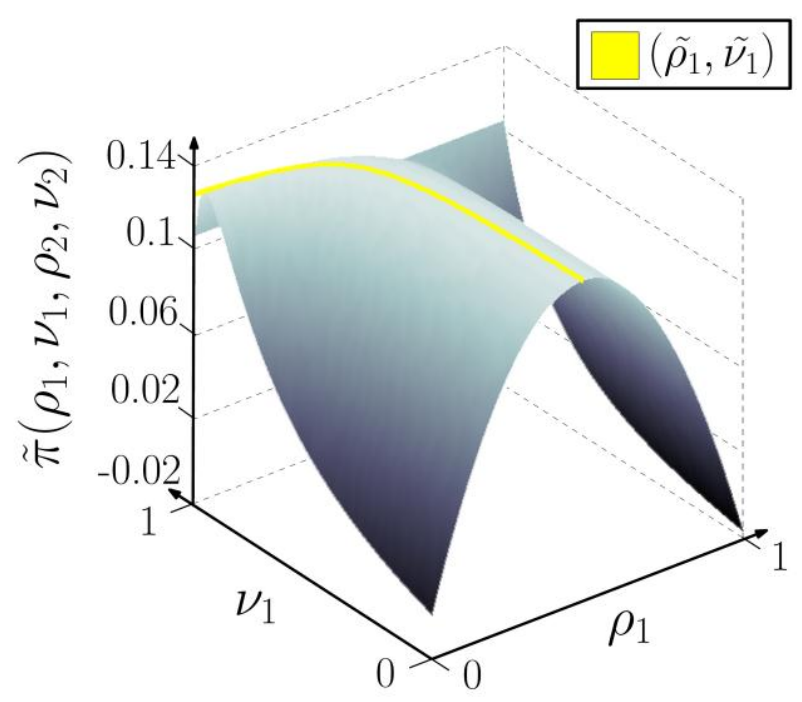

Figure 7: An example of the payoff function of one player given the other player's strategy $\left(\rho_{2}=0.7\right.$ and $\left.v_{2}=0.9\right)$.

The Nash equilibrium $\left(\rho_{1}^{\#}, v_{1}^{\#}, \rho_{2}^{\#}, v_{2}^{\#}\right)$, consists of all possible outcomes such that the strategy of each player is a best response to the other player's strategy. Thus this includes all such points where

$$
\arg \max _{\rho_{j}, v_{j}} \tilde{\pi}_{i}\left(\rho_{i}, v_{i}, \rho_{j}, v_{j}\right)=\arg \max _{\rho_{i}, v_{i}} \tilde{\pi}_{j}\left(\rho_{j}, v_{j}, \rho_{i}, v_{i}\right), i \in I
$$

Solving this condition algebraically gives the following description of the Nash equilibria

$$
\begin{aligned}
& \rho_{1}^{\#}=\frac{v_{1}^{\#}\left(3 v_{2}^{\#}-v_{1}^{\#}-2 v_{1}^{\#} v_{2}^{\#}\right)}{\left(1-v_{1}^{\#}\right)\left(v_{1}^{\#}+v_{2}^{\#}+2 v_{1}^{\#} v_{2}^{\#}\right)}, \\
& \rho_{2}^{\#}=\frac{v_{2}^{\#}\left(3 v_{1}^{\#}-v_{2}^{\#}-2 v_{1}^{\#} v_{2}^{\#}\right)}{\left(1-v_{2}^{\#}\right)\left(v_{1}^{\#}+v_{2}^{\#}+2 v_{1}^{\#} v_{2}^{\#}\right)},
\end{aligned}
$$

which shows that there exist an infinite number of Nash equilibria in this formulation of the consumption game.

\subsection{Game tragicness}

In the consumption game $G$, the optimal strategy $\left(\rho_{1}^{*}, \rho_{2}^{*}\right)$ maximises 


$$
J\left(\rho_{1}, \rho_{2}\right)=\pi_{1}\left(\rho_{1}, \rho_{2}\right)+\pi_{2}\left(\rho_{1}, \rho_{2}\right)
$$

Thus,

$$
J\left(\rho_{1}^{*}, \rho_{2}^{*}\right)=\max _{\rho_{1}, \rho_{2}} J\left(\rho_{1}, \rho_{2}\right) .
$$

By calculating the partial derivatives and putting them to zero, we find that for any $v_{1}, v_{2}, J(\cdot, \cdot)$ is maximized not at a point, but rather along a curve, which is given by

$$
2 v_{2}\left(1-v_{1}\right) \rho_{1}^{*}+2 v_{1}\left(1-v_{2}\right) \rho_{2}^{*}-v_{1}\left(1-v_{2}\right)-v_{2}\left(1-v_{1}\right)=0 .
$$

A single realization of this curve is shown in Figure 8. A "non-tragic" game is one in which the Nash equilibrium lies exactly on this optimal curve. A game, in which the Nash equilibrium does not lie on this curve, is a "tragic" one.

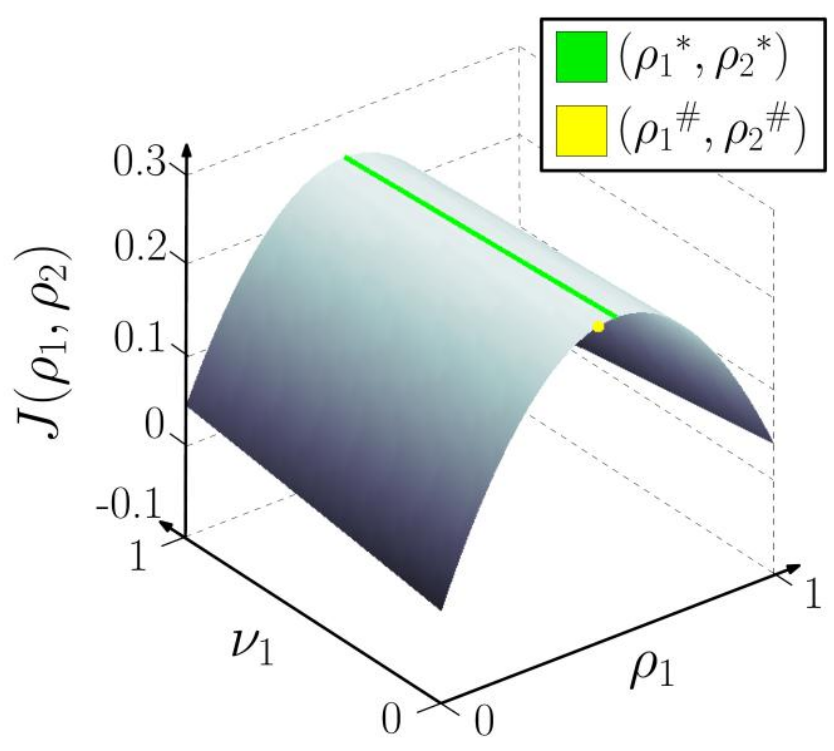

Figure 8: An example of the optimal strategy curve $\left(v_{1}=0.3\right.$ and $\left.v_{2}=0.9\right)$.

We introduce the tragicness of $G$ as the Euclidean norm of the shortest line joining the Nash equilibrium given by (10) and the optimal curve given by (11), which is given as

$$
\text { Tragicness }=\frac{\left|4 v_{1} v_{2} \frac{v_{1}\left(1-v_{2}\right)+v_{2}\left(1-v_{1}\right)}{v_{1}\left(1+v_{2}\right)+v_{2}\left(1+v_{1}\right)}-v_{1}\left(1-v_{2}\right)-v_{2}\left(1-v_{1}\right)\right|}{4 v_{2}^{2}\left(1-v_{1}\right)^{2}-4 v_{1}^{2}\left(1-v_{2}\right)^{2}} .
$$

